J. Dairy Sci. 92:758-764

doi:10.3168/jds.2008-1395

(c) American Dairy Science Association, 2009.

\title{
Fine mapping and association analysis of a quantitative trait locus for milk production traits on Bos taurus autosome 4
}

\author{
G. Rincón, * A. Islas-Trejo, * J. Casellas,† Y. Ronin,‡ M. Soller,§ E. Lipkin,§ and J. F. Medrano*1 \\ *Department of Animal Science, University of California, Davis 95616 \\ †Genètica i Millora Animal, Institut de Recerca i Tecnologia Agroalimentaries-Lleida, 25198 Lleida, Spain \\ łInstitute of Evolution, University of Haifa, Mount Carmel, Haifa 31905, Israel \\ $\S$ Department of Genetics, The Hebrew University of Jerusalem, 91904 Jerusalem, Israel
}

\begin{abstract}
To fine map a quantitative trait locus (QTL) affecting milk production traits previously associated with microsatellite RM188, we implemented an interval mapping analysis by using microsatellite markers in a large Israeli Holstein half-sib sire family, and linkage disequilibrium (LD) mapping in a large set of US Holstein bulls. Interval mapping located the target QTL to the near vicinity of RM188. For the LD mapping, we identified 42 single nucleotide polymorphisms (SNP) in 15 genes in a $12-\mathrm{Mb}$ region on bovine chromosome 4 . A total of 24 tag SNP were genotyped in 882 bulls belonging to the University of California Davis archival collection of Holstein bull DNA samples with predicted transmitted ability phenotypes. Marker-to-marker LD analysis revealed 2 LD blocks, with intrablock $\mathrm{r}^{2}$ values of 0.10 and 0.46 , respectively; outside the blocks, $\mathrm{r}^{2}$ values ranged from 0.002 to 0.23 . A standard additive/ dominance model using the generalized linear model procedure of SAS and the regression module of HelixTree software were used to test marker-trait associations. Single nucleotide polymorphism 9 on ARL4A, SNP10 on XR_027435.1, SNP12 on ETV1, SNP21 on SNX13, and SNP24 were significantly associated with milk production traits. We propose the interval encompassing ARL4A and SNX13 genes as a candidate region in bovine chromosome 4 for a concordant QTL related to milk protein traits in dairy cattle. Functional studies are needed to confirm this result.
\end{abstract}

Key words: Bos taurus autosome 4, milk trait quantitative trait locus, association analysis, microsatellite RM188

\section{INTRODUCTION}

Several genome-wide scans for QTL in dairy cattle have provided primary localizations of numerous QTL

Received May 26, 2008.

Accepted September 30, 2008.

${ }^{1}$ Corresponding author: jfmedrano@ucdavis.edu affecting production (milk yield, fat yield and percentage, and protein yield and percentage), health, and conformational traits. Results have recently been reviewed by Khatkar et al. (2004) and can be accessed in QTL databases (Polineni et al., 2006; Hu et al., 2007). The ultimate goal of QTL mapping is to identify the underlying functional elements corresponding to the QTL. These are generally referred to as quantitative trait genes (QTG), although they may include functional sequences of any type. Identification of QTG and their function is important to understand the nature of their effect on phenotypic variation in milk production traits. In addition, genotypic information on the functional mutation at the QTG affecting traits of interest, or identification of markers or marker haplotypes closely linked to the functional mutation, can be used either in direct selection on functional genes or in markerassisted selection.

The positional information provided by standard QTL mapping procedures in dairy cattle has wide confidence intervals and does not provide sufficiently narrow positional information for effective identification of candidate genes for association tests (Ronin et al., 2003; Weller and Soller, 2004). Recently, it has been shown that combining linkage interval mapping and linkage disequilibrium (LD) mapping by using a dense SNP marker map can effectively reduce the confidence intervals of QTL locations to a few centimorgans or less, in this way greatly facilitating QTG identification (Georges, 2007; Solberg et al., 2008).

Mosig et al. (2001) used QTL mapping by selective DNA pooling to screen bovine autosomes for QTL affecting EBV for milk protein percentage (EBVP\%). In that study, the microsatellite marker RM188 was found to be strongly associated with EBVP\% $(P<0.033$; false discovery rate $<0.05)$. Earlier, the same region was found linked to Weaver disease and loosely linked to milk production (Georges et al., 1993; Medjugorac et al., 1996). Further studies in our laboratory, using selective genotyping in a very large single Israeli Holstein sire sibship, confirmed the associated effect of RM188 with EBVP\% and showed an even stronger associated 
effect on EBV for protein yield $(\mathrm{kg} / \mathrm{yr})$. On this basis, this region was chosen for high-resolution QTL mapping by interval mapping using microsatellite markers, and by an LD association test focusing on SNP markers across this region. Thus, this study can be considered a combined LDLA analysis, with the linkage component (LA) and LD component based on completely independent samples, and hence being strongly supportive.

\section{MATERIALS AND METHODS}

\section{Linkage Interval Mapping}

Interval mapping of the QTL associated with RM188 was based on the Israeli Holstein sire "Scorer," having more than 9,000 milking daughters. Marker-QTL analysis for EBV for milk protein yield (EBVPY) by selective DNA pooling indicated a strong QTL affecting this trait in the proximal region of Bos taurus autosome (BTA) 4, near marker RM188 (data not shown), confirming the previous results of Mosig et al. (2001). To locate the QTL more precisely, 780 daughters from the greatest $10 \%$ of all daughters of this sire with respect to EBVPY, and an equal number from the $10 \%$ of lowest daughters for this trait were individually genotyped for 4 markers spanning $32 \mathrm{cM}$ on the proximal region of BTA4: BMS1788 at $12.5 \mathrm{cM}$; RM188 at 28.4 $\mathrm{cM}$; BMS1237 at $34.4 \mathrm{cM}$; and BMS2646 at $43.2 \mathrm{cM}$ (USDA bovine genome map, http://www.marc.usda. gov/genome/cattle/cattle.html). Genotyping was as described previously (Lipkin et al., 1998; Mosig et al., 2001). Interval mapping analysis was performed using the Multi-QTL package (http://www.multiqtl.com/).

\section{LD Mapping}

Animals. Gene resequencing was performed in the 8 Israeli Holstein bulls that were the progenitors segregating the QTL in the population where the QTL region was identified (Mosig et al., 2001 and our unpublished data). The samples used for the association study consisted of 882 Holstein bulls from the University of California Davis archival collection begun in 1995 and consisting of approximately 1,100 bull DNA samples extracted from frozen semen straws obtained from commercial AI companies. The average sire family size was 8 , and the number of grandsires represented in the bull sample population was 146 .

Traits. Predicted transmitting ability for milk, fat, protein, fat percentage, protein percentage, cheese dollars, and net merit (NM) dollar values were obtained from the USDA Animal Improvement Programs Laboratory (Beltsville, MD; available at http://aipl.arsusda. gov/eval.htm). Average reliabilities for the PTA and
NM traits used in the association analysis were 93.06 and $87.80 \%$, respectively.

\section{SNP Discovery and Genotyping}

The fine mapping of QTL affecting milk production traits on BTA4 covered a $12-\mathrm{Mb}$ region flanking microsatellite RM188. The fine-mapping strategy consisted of identifying primary candidate genes based on location or physiological relevance to the trait. Gene expression profiles for all target genes were obtained from our bovine mammary gland expression database based on the Affymetrix GeneChip Bovine Genome Array (our unpublished data). Single nucleotide polymorphisms in gene regions were identified by using the dbSNP build 128 from the National Center for Biotechnology Information database (http://www.ncbi.nlm.nih.gov/SNP/ index.html) and the Interactive Bovine In-Silico SNP Database from the Commonwealth Scientific and Industrial Research Organisation (http://www.livestockgenomics.csiro.au/cattle.shtml).

To narrow the target region and to confirm the gene order and annotation in the bovine genome, we used a comparative mapping approach combining bovine microsatellite information mapped to the human, mouse, and dog genome assemblies, as described by Farber and Medrano (2003). The target genomic regions were resequenced in the 8 Israeli Holstein founder bulls to confirm the presence of polymorphism found in databases in the Holstein breed, to discover new SNP, and to analyze informativity.

Primers were designed by using Primer3 software (http://frodo.wi.mit.edu/) to generate primer sets for each target genomic region (Table 1). The PCR amplifications were performed at an annealing temperature of $60^{\circ} \mathrm{C}$, with $2 \mathrm{mM} \mathrm{MgCl} 2$ and $0.7 \mathrm{U}$ of AmpliTaq DNA polymerase (Applied Biosystems, Foster City, CA). Polymerase chain reaction fragments were gel purified by using Promega Wizard SV gel and the PCR Cleanup System and resequenced at SeqWright DNA Technology Services (Houston, TX). More than 600 traces were generated and assembled by using the CodonCode Aligner (http://www.codoncode.com/). Each SNP was confirmed in amplicons sequenced in the forward and reverse directions. In total, $42 \mathrm{SNP}$ were identified in the RM188 flanking region. Twenty-four SNP were selected for genotyping and association analysis based on 3 criteria: 1) location on BTA4 related to the position of RM188, 2) position on coding genes in the region (i.e., exons, promoter regions, conserved regions), and 3) genotypic information from the 8 Israeli Holstein founder bulls.

Deoxyribonucleic acid samples $(\mathrm{n}=882)$ were genotyped, for a total of 24 SNP (Table 1), by using the Se- 
Table 1. Forty-two SNP in Bos taurus autosome 4 (BTA4) flanking microsatellite RM188 that were identified by resequencing: locus, source, National Center for Biotechnology Information (NCBI) identification (ID), location, and PCR primers used to amplify the resequenced amplicon $^{1}$

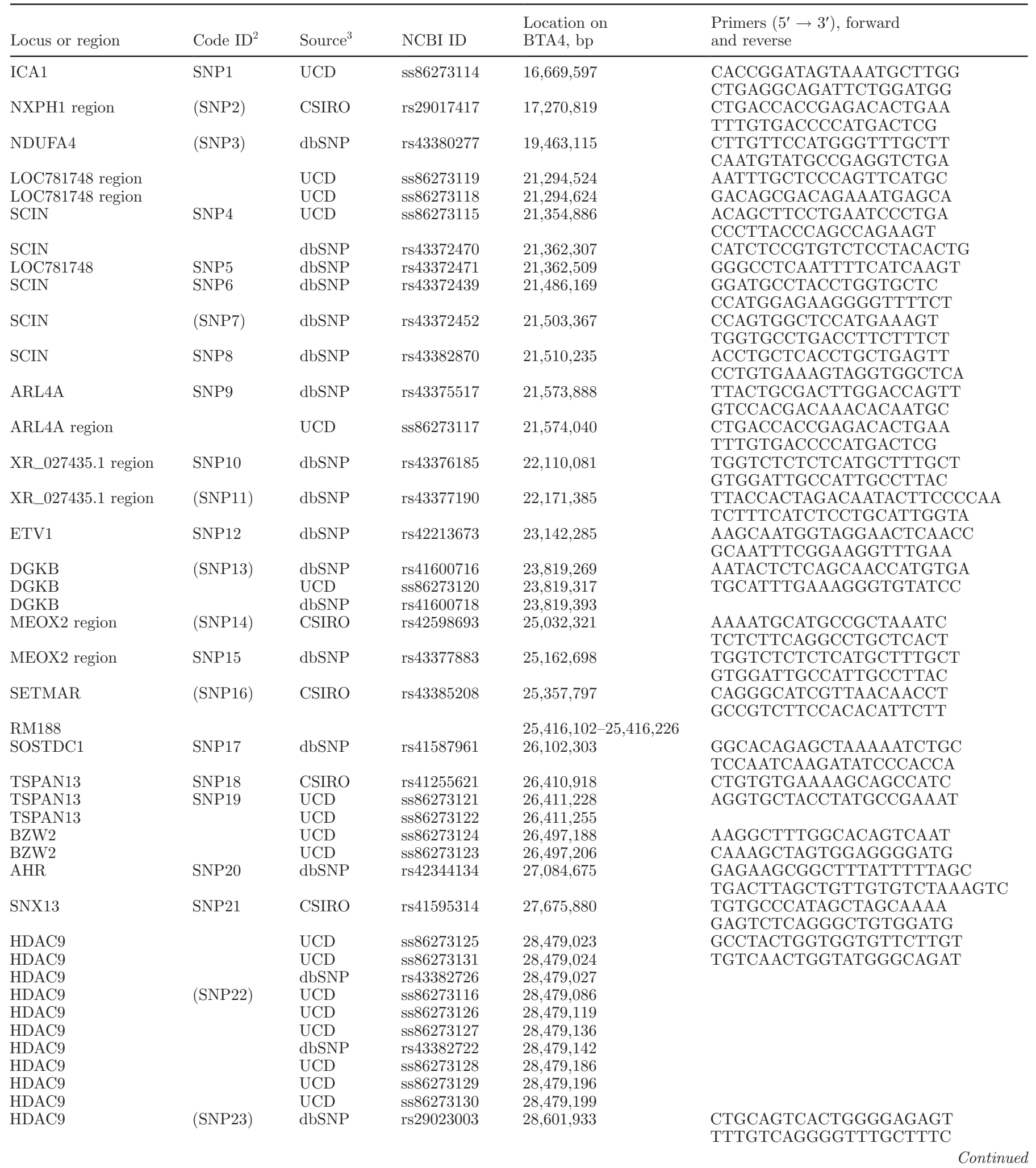


Table 1 (Continued). Forty-two SNP in Bos taurus autosome 4 (BTA4) flanking microsatellite RM188 that were identified by resequencing: locus, source, National Center for Biotechnology Information (NCBI) identification (ID), location, and PCR primers used to amplify the resequenced amplicon ${ }^{1}$

\begin{tabular}{|c|c|c|c|c|c|}
\hline Locus or region & Code ID $^{2}$ & Source $^{3}$ & NCBI ID & $\begin{array}{l}\text { Location on } \\
\text { BTA } 4 \text {, bp }\end{array}$ & $\begin{array}{l}\text { Primers }\left(5^{\prime} \rightarrow 3^{\prime}\right), \text { forward } \\
\text { and reverse }\end{array}$ \\
\hline SNP19 & SNP24 & dbSNP & rs43745957 & & $\begin{array}{l}\text { GGGCTCACACGGTAAAGAAT } \\
\text { GAGGTCCTACTTCTCCCTCCA }\end{array}$ \\
\hline
\end{tabular}

${ }^{1}$ SNP locations are according to Bos taurus assembly BTA4.0.

${ }^{2} \mathrm{SNP}$ used for genotyping and association analyses were numbered 1 to 24 relative to location; SNP not used for marker-to-marker linkage disequilibrium and marker-trait association analyses are in parentheses.

${ }^{3} \mathrm{UCD}=\mathrm{SNP}$ identified at University of California Davis; CSIRO = SNP from the Interactive Bovine In-Silico SNP Database from the Commonwealth Scientific and Industrial Research Organisation; dbSNP = SNP from the Single Nucleotide Polymorphism database of the National Center for Biotechnology Information. All SNP were confirmed by resequencing. Single nucleotide polymorphism 19 was located on Contig115195 at 6,838 bp.

quenom MassARRAY platform at GeneSeek Inc. (Lincoln, NE). The Hardy-Weinberg equilibrium (HWE) test was performed for each marker by using GENEPOP software version 3.1c (Rousset and Raymond, 1995). Single nucleotide polymorphism 3, SNP7, and SNP14 (Table 1) were not in the HWE; the genotyping essays did not work for SNP2, SNP11, SNP13, SNP16, SNP22, and SNP23. Although SNP24 had several regions of homology along the genome, it was positioned on BTA4 based on $r^{2}$ values, as described by (Miller et al., 2006). Thus, 15 SNP remained (SNP1, SNP4, SNP5, SNP6, SNP8, SNP9, SNP10, SNP12, SNP15, SNP17, SNP18, SNP19, SNP20, SNP21, and SNP24), which were used for analysis of marker-to-marker LD, and for marker-trait association tests.

\section{LD and Association Analysis}

Marker-to-marker LD analysis was performed by using the software Haploview (http://www.broad.mit. $\mathrm{edu} / \mathrm{mpg} /$ haploview/). The parameters used to run the LD analysis in Haploview software were as follows: minimum genotyped percentage, 75; Hardy-Weinberg $P$-value cutoff, 0.010; minimum minor allele frequency, 0.010 .

Marker-trait association analyses were performed on the milk traits listed above for the University of California Davis bull population. Normality of phenotypic data was tested with the univariate procedure of SAS (SAS Inst. Inc., Cary, NC). Association analyses were performed independently on each SNP and under a standard additive/dominance model (Falconer, 1960) by using the GLM procedure of SAS (SAS Inst. Inc.),

$$
y_{i j}=\mu+a_{i}+d_{i}+e_{i j},
$$

where $y_{i j}$ was a phenotypic record influenced by the overall mean $(\mu)$ as well as the additive $\left(a_{i}\right)$ and domi- nant effect $\left(d_{i}\right)$ of individual $i$, and $e_{i j}$ was the residual term.

The association analysis was also performed by using the regression module from Helixtree software version 6.3.1 (Golden Helix Inc., Bozeman, MT). Because PTA values are intrinsically additive, the expectation was that the GLM analysis would uncover significant additive effects, but that dominance effects would not be significant. The regression module of the Helixtree software corresponded to the additive allele substitution model. For a purely additive genetic situation, this model should provide greater power than the GLM. The allele substitution model has been used successfully in the past for analysis of data of this type (Khatib et al., 2005, 2006; Cobanoglu et al., 2006). Therefore, we believe that the results obtained with the Helixtree regression module best represent the actual data model. Because this is a multiple-test situation, false discovery rate was calculated for the analysis by using the Helixtree algorithm.

\section{RESULTS AND DISCUSSION}

Interval mapping based on the individual genotyping data for the sire Scorer gave a maximum logarithm of the odds (LOD) at the location of RM188 itself, with a $95 \%$ confidence interval of QTL location by bootstrap analysis extending $9.5 \mathrm{cM}$ to either side. Significance by the permutation test was at the $P=0.002$ level. To further define the location of the QTL with respect to RM188, marker allele-associated effects on EBVPY of daughters that were recombinant or nonrecombinant across the RM188 region (from BMS1788 to BMS2646) were compared. The mean difference between daughters receiving one or the other of the 2 sire alleles ( 4.3 $\mathrm{kg} / \mathrm{yr}$ ) was exactly the same for recombinant and nonrecombinant daughters. Because recombination would be expected to attenuate the difference between sire 
marker alleles, this indicates that the QTL was very closely linked to RM188.

Based on the interval mapping results, we examined candidate genes to fine map a QTL affecting milk production traits associated with microsatellite RM188. Fifteen candidate genes flanking the RM188 region were screened for SNP, and 42 SNP were identified in a $12-\mathrm{Mb}$ region on BTA4. All the SNP were mapped to bovine genome assembly BTA3.1 and to the new assembly BTA4.0 (B. taurus linear scaffolds as of Sep. 13, 2007). Table 1 shows the location, source, and dbSNP identification of the 42 SNP. The SNP selected for genotyping and association analyses are shown as SNP1 to SNP24. In most cases, the order of the SNP from assembly BTA3.1 to BTA4.0 remained the same. Some exceptions were 1) SNP5 was identified in BTA3.1 next to SNP9, but in BTA4.0 it appeared in the same scaffold together with the SCIN gene; b) SNP16 was relocated in BTA4.0 within intron 4 of the SETMAR gene; and 3) SNP24 had several hits along the genome, and it was not possible to ascertain an accurate location. The LD between markers was used to infer the position of SNP24 in the new assembly BTA4.0, as described by Miller et al. (2006). Based on the $\mathrm{r}^{2}$ values, the most likely position for SNP24 is between SNP12 and SNP15, corresponding to genes ETV1 and MEOX2, respectively. The location of SNP24 was also confirmed by using the MegaBLAST B. taurus option from the Center for Bioinformatics and Computational Biology, University of Maryland (http://blast.cbcb. umd.edu/megablast.html), which located SNP24 on chromosome 4 at 24,736,211 bp between the ETV1 and MEOX2 genes.

Marker-to-marker LD analysis and marker-trait association analyses were based on genotypes from the 15 retained SNP in 882 individual samples. Two LD blocks were clearly identified by using $\mathrm{r}^{2}$ statistics (Figure 1).
The first LD block corresponded to SNP4 and SNP5 $\left(\mathrm{r}^{2}\right.$ $=0.10$ ). The second LD block corresponded to SNP18 and SNP19 $\left(\mathrm{r}^{2}=0.46\right)$. Outside the blocks, $\mathrm{r}^{2}$ values ranged from 0.002 to 0.23 . The region flanking RM188 (SNP10, SNP12, SNP15, and SNP16) showed the lowest linkage disequilibrium coefficient $\left(\mathbf{D}^{\prime}\right)$ and $r^{2}$ values and may represent a recombination "hot spot."

Recently, Khatkar et al. (2008) performed a comprehensive study of the extent of LD in cattle by analyzing data on 1,546 Holstein-Friesian bulls genotyped for 15,036 SNP markers. They examined the minimum sample sizes required to estimate $\mathrm{LD}$ without bias and loss of accuracy. Significant LD in cattle extended to $40 \mathrm{~kb}$ when estimated as $\mathrm{r}^{2}$. For estimation of LD by $\mathrm{D}^{\prime}$ with sufficient precision, a sample size of at least 400 individuals was required, whereas for $\mathrm{r}^{2}$, a minimum sample of 75 was adequate (Khatkar et al., 2008). Thus, the marker-to-marker LD values obtained in this analysis can be taken as representative.

The association analysis using a GLM model gave significant associations $(P \leq 0.05)$ for SNP9, SNP10, SNP12, SNP21, and SNP24 and the traits PTA for milk (lb), PTA for protein (lb), PTA for protein percentage (\%), cheese dollars (\$), and NM dollars (Table 2). A comparable association analysis was performed by using the regression analysis module of Helixtree. This software was developed for SNP-based complex candidate gene and whole-genome association studies and has been largely used in human studies (Barton et al., 2004; Carlson et al., 2004; Hinks et al., 2006; Lencz et al., 2007). The Helixtree software has novel analytical and graphical tools that we felt were worthwhile to examine for association analysis in livestock in comparison with conventional GLM procedures.

The results obtained with the GLM procedure (Table 2 ) showed that only the additive effects were significant; none of the dominance effects was significant, and most

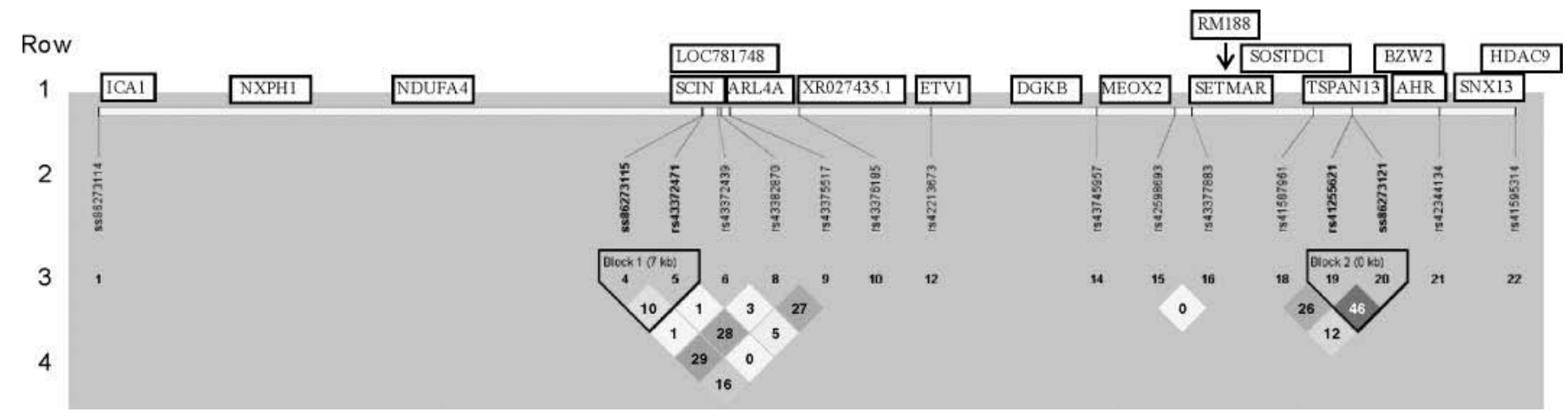

Figure 1. Linkage disequilibrium (LD) map of SNP in Hardy-Weinberg equilibrium for the University of California Davis bull population. Row 1, locus name; row 2, SNP National Center for Biotechnology Information identification; row 3, Haploview SNP identification; row 4, LD blocks. Dark squares represent high $\mathrm{r}^{2}$ values, and triangles surrounding markers represent haplotype blocks under the 4-gamete rule (Wang et al., 2002). Numbers in squares are $\mathrm{r}^{2}$ values. 
Table 2. Significant SNP associated with milk traits in the University of California Davis bull population by using a standard additive/ dominance model with the GLM procedure of $\mathrm{SAS}^{1}$ and using Helixtree regression module ${ }^{2}$

\begin{tabular}{|c|c|c|c|c|c|c|c|c|c|c|}
\hline \multirow[b]{2}{*}{ Marker } & \multirow{2}{*}{$\begin{array}{l}\text { SNP }^{3} \\
\text { MAF }^{3}\end{array}$} & \multicolumn{7}{|c|}{ GLM } & & \\
\hline & & Trait $^{4}$ & Estimate & $\mathrm{SE}$ & $P$-value & Estimate & $\mathrm{SE}$ & $P$-value & $P$-value & False discovery rate \\
\hline SNP9 & 0.46 & PTAM, lb & 87.12 & 35.48 & 0.014 & -12.88 & 50.05 & 0.796 & 0.020 & $1.89 \mathrm{E}-01$ \\
\hline \multirow[t]{2}{*}{ SNP10 } & 0.34 & PTAM, lb & -87.98 & 39.94 & 0.028 & -93.20 & 54.06 & 0.085 & 0.008 & $1.06 \mathrm{E}-02$ \\
\hline & & PTAPP, $\%$ & 0.77 & 0.24 & 0.002 & 0.29 & 0.33 & 0.387 & 0.0003 & 4.01E-03 \\
\hline & & PTAP, lb & 2.97 & 1.77 & 0.095 & 0.80 & 2.109 & 0.702 & 0.003 & $2.29 \mathrm{E}-02$ \\
\hline SNP21 & 0.12 & PTAPP, $\%$ & -1.58 & 0.73 & 0.031 & 1.05 & 0.80 & 0.191 & 0.014 & $1.80 \mathrm{E}-01$ \\
\hline \multirow{2}{*}{ SNP24 } & 0.36 & CheeseD, $\$$ & -27.37 & 10.605 & 0.010 & -4.41 & 14.26 & 0.757 & 0.010 & $1.40 \mathrm{E}-01$ \\
\hline & & NMD, $\$$ & -28.97 & 10.386 & 0.005 & -4.75 & 13.96 & 0.733 & 0.008 & $7.10 \mathrm{E}-02$ \\
\hline
\end{tabular}

${ }^{1}$ SAS Institute Inc. (Cary, NC).

${ }^{2}$ Significance level, $P \leq 0.05$; false discovery rate $\leq 0.20$.

${ }^{3}$ Single nucleotide polymorphism minor allele frequency (MAF).

${ }^{4} \mathrm{PTAM}=\mathrm{PTA}$ for milk; PTAPP $=$ PTA for protein percentage; CheeseD = cheese dollars; NMD = net merit dollars; PTAP $=$ PTA for protein.

were far from significance. Thus, this analysis confirmed the basically additive nature of the PTA and NM traits, as expected, and justifies use of the Helixtree regression module for analysis of these data. The $P$-values obtained by the Helixtree regression module (Table 2) were generally comparable, although they were often lower than those provided by the GLM analysis. This is reasonable because, as noted, the regression analysis is more suitable to a purely additive situation.

The results observed in both analyses indicated that the major effects on milk traits were observed for SNP9, SNP10, SNP12, SNP21, and SNP24. Single nucleotide polymorphism 9 is located on the ARL4A $5^{\prime}$ untranslated region; SNP10 is an intergenic SNP located in proximity to the XR_027435.1 gene; SNP12 is on ETV1 intron 6; SNP21 is located on sorting nexin (SNX) 13 intron 3; SNP24 is intergenic and is located in proximity to MEOX2 gene. The ARL4A is a highly conserved protein that participates in exocytic and endocytic vesicular transport pathways in different cell compartments (Jacobs et al., 1999). The ETV1 gene encodes a protein with transcription factor activity and sequence-specific DNA-binding properties localized in the mitochondrion and nucleus. This gene has been tested for association with carcinoma, prostatic intraepithelial neoplasia, prostatic neoplasms, and sarcoma (Dowdy et al., 2003; Cerveira et al., 2006). The SNX13 gene encodes a PHOX domain- and RGS domain-containing protein that belongs to the SNX family involved in intracellular trafficking (Worby and Dixon, 2002). The MEOX2 proteins are localized in the nucleus and participate in sequence-specific DNA-binding and transcription factor activity (Chen et al., 2007). Although we did not use any SNP located on the MEOX2 gene for the association analysis, the location, the high level of expression in the lactating mammary gland, and the proximity to SNP24 associated with milk traits make it a target candidate gene in the QTL region. Based on our bovine mammary gland expression studies using the bovine Affymetrix gene chip (our unpublished data), genes ARL4A and MEOX2 are highly expressed in the bovine mammary gland during lactation. There are no mRNA probes for XR_027435.1 and SNX13 in the Affymetrix chip, and there is no evidence for the expression of the ETV1 gene. However, based on gene expression information obtained from the mouse and human databases BioGPS (https://biogps.gnf. org/) and Gene Expression Omnibus profiles (http:// www.ncbi.nlm.nih.gov/projects/geo/info/mouse-trans. html), SNX13 is expressed to some degree in mammary tissue, whereas ETV1 is not expressed in the mammary gland.

None of the SNP that were significantly associated with milk production traits in this study encoded a nonconservative AA substitution. As observed in the majority of the quantitative trait nucleotides identified in livestock, the expectation that a polymorphism associated with a trait would reside in sequences encoding AA substitutions has not been realized (Ron and Weller, 2007). These results support the importance of regulatory mutations located on introns or intergenic regions controlling phenotypic variation.

In the present study, 2 large and independent samples from 2 different Holstein populations, one based on classical linkage analysis and the other on LD analysis, each confirmed previous results based on selective 
DNA pooling, and strongly support the region tightly linked to RM188 as a candidate region for a QTL having a major effect on milk production traits. The LD association test results further indicate that ARL4A, XR_027435.1, ETV1, MEOX2, and SNX13 are attractive candidate genes for further detailed studies in relation to milk production traits. Additional research will be needed to clarify the role of significantly associated SNP in the functional mechanisms of each milk production trait.

\section{ACKNOWLEDGMENTS}

This work was supported by United States-Israel Binational Agricultural Research and Development Fund (BARD) Project No. US-3406-03 R, and by The California Dairy Research Foundation Project No. 05 MEJ-01-NH.

\section{REFERENCES}

Barton, A., J. A. Woolmore, D. Ward, S. Eyre, A. Hinks, W. E. Ollier, R. C. Strange, A. A. Fryer, S. John, C. P. Hawkins, and J. Worthington. 2004. Association of protein kinase C alpha (PRKCA) gene with multiple sclerosis in a UK population. Brain 127:1717-1722.

Carlson, C. S., M. A. Eberle, M. J. Rieder, Q. Yi, L. Kruglyak, and D. A. Nickerson. 2004. Selecting a maximally informative set of single-nucleotide polymorphisms for association analyses using linkage disequilibrium. Am. J. Hum. Genet. 74:106-120.

Cerveira, N., F. R. Ribeiro, A. Peixoto, V. Costa, R. Henrique, C. Jeronimo, and M. R. Teixeira. 2006. TMPRSS2-ERG gene fusion causing ERG overexpression precedes chromosome copy number changes in prostate carcinomas and paired HGPIN lesions. Neoplasia 8:826-832.

Chen, Y., A. D. Leal, S. Patel, and D. H. Gorski. 2007. The homeobox gene GAX activates p21WAF1/CIP1 expression in vascular endothelial cells through direct interaction with upstream AT-rich sequences. J. Biol. Chem. 282:507-517.

Cobanoglu, O., I. Zaitoun, Y. M. Chang, G. E. Shook, and H. Khatib. 2006. Effects of the signal transducer and activator of transcription 1 (STAT1) gene on milk production traits in Holstein dairy cattle. J. Dairy Sci. 89:4433-4437.

Dowdy, S. C., A. Mariani, and R. Janknecht. 2003. HER2/Neu- and TAK1-mediated up-regulation of the transforming growth factor beta inhibitor Smad7 via the ETS protein ER81. J. Biol. Chem. 278:44377-44384.

Farber, C. R., and J. F. Medrano. 2003. Putative in silico mapping of DNA sequences to livestock genome maps using SSLP flanking sequences. Anim. Genet. 34:11-18.

Georges, M. 2007. Mapping, fine mapping, and molecular dissection of quantitative trait loci in domestic animals. Annu. Rev. Genomics Hum. Genet. 8:131-162.

Georges, M., A. B. Dietz, A. Mishra, D. Nielsen, L. S. Sargeant, A. Sorensen, M. R. Steele, X. Zhao, H. Leipold, and J. E. Womack 1993. Microsatellite mapping of the gene causing Weaver disease in cattle will allow the study of an associated quantitative trait locus. Proc. Natl. Acad. Sci. USA 90:1058-1062.

Hinks, A., A. Barton, S. John, N. Shephard, and J. Worthington. 2006. Fine mapping of genes within the IDDM8 region in rheumatoid arthritis. Arthritis Res. Ther. 8:R145.

Hu, Z. L., E. R. Fritz, and J. M. Reecy. 2007. AnimalQTLdb: A livestock QTL database tool set for positional QTL information mining and beyond. Nucleic Acids Res. 35(Database issue):D604D609.

Jacobs, S., C. Schilf, F. Fliegert, S. Koling, Y. Weber, A. Schurmann, and H. G. Joost. 1999. ADP-ribosylation factor (ARF)-like 4, 6 , and 7 represent a subgroup of the ARF family characterization by rapid nucleotide exchange and a nuclear localization signal. FEBS Lett. 456:384-388.

Khatib, H., E. Heifetz, and J. C. Dekkers. 2005. Association of the protease inhibitor gene with production traits in Holstein dairy cattle. J. Dairy Sci. 88:1208-1213.

Khatib, H., S. D. Leonard, V. Schutzkus, W. Luo, and Y. M. Chang. 2006. Association of the OLR1 gene with milk composition in Holstein dairy cattle. J. Dairy Sci. 89:1753-1760.

Khatkar, M. S., F. W. Nicholas, A. R. Collins, K. R. Zenger, J. A. Cavanagh, W. Barris, R. D. Schnabel, J. F. Taylor, and H. W. Raadsma. 2008. Extent of genome-wide linkage disequilibrium in Australian Holstein-Friesian cattle based on a high-density SNP panel. BMC Genomics 9:187.

Khatkar, M. S., P. C. Thomson, I. Tammen, and H. W. Raadsma. 2004. Quantitative trait loci mapping in dairy cattle: Review and meta-analysis. Genet. Sel. Evol. 36:163-190.

Lencz, T., C. Lambert, P. DeRosse, K. E. Burdick, T. V. Morgan, J. M. Kane, R. Kucherlapati, and A. K. Malhotra. 2007. Runs of homozygosity reveal highly penetrant recessive loci in schizophrenia. Proc. Natl. Acad. Sci. USA 104:19942-19947.

Lipkin, E., M. O. Mosig, A. Darvasi, E. Ezra, A. Shalom, A. Friedmann, and M. Soller. 1998. Quantitative trait locus mapping in dairy cattle by means of selective milk DNA pooling using dinucleotide microsatellite markers: Analysis of milk protein percentage. Genetics 3:1557-1567.

Medjugorac, I., I. Russ, J. Aumann, and M. Förster. 1996. Weaver carrier status effects on yield in German Brown cattle. Anim. Genet. 27(Suppl.):105.

Miller, S. P., B. J. Hayes, and M. E. Goddard. 2006. Positioning single nucleotide polymorphisms on an existing bovine map using a genetic algorithm and estimates of linkage disequilibrium. Session 21, no. 14 (Linkage disequilibrium mapping) in 8th World Congr. Genet. Appl. Livest. Prod., Belo Horizonte, Minas Gerais, Brazil.

Mosig, M. O., E. Lipkin, G. Khutoreskaya, E. Tchourzyna, M. Soller, and A. Friedmann. 2001. A whole genome scan for quantitative trait loci affecting milk protein percentage in Israeli-Holstein cattle, by means of selective milk DNA pooling in a daughter design, using an adjusted false discovery rate criterion. Genetics 157:1683-1698.

Polineni, P., P. Aragonda, S. R. Xavier, R. Furuta, and D. L. Adelson. 2006. The bovine QTL viewer: A web accessible database of bovine quantitative trait loci. BMC Bioinformatics 7:283.

Ron, M., and J. I. Weller. 2007. From QTL to QTN identification in livestock-Winning by points rather than knock-out: A review. Anim. Genet. 38:429-439.

Ronin, Y., A. Korol, M. Shtemberg, E. Nevo, and M. Soller. 2003. High-resolution mapping of quantitative trait loci by selective recombinant genotyping. Genetics 164:1657-1666.

Rousset, F., and M. Raymond. 1995. Testing heterozygote excess and deficiency. Genetics 140:1413-1419.

Solberg, T. R., A. K. Sonesson, J. A. Woolliams, and T. H. Meuwissen. 2008. Genomic selection using different marker types and densities. J. Anim. Sci. 86:2447-2454.

Wang, N., J. M. Akey, K. Zhang, R. Chakraborty, and L. Jin. 2002. Distribution of recombination crossovers and the origin of haplotype blocks: The interplay of population history, recombination, and mutation. Am. J. Hum. Genet. 71:1227-1234.

Weller, J. I., and M. Soller. 2004. An analytical formula to estimate confidence interval of QTL location with a saturated genetic map as a function of experimental design. Theor. Appl. Genet. 109:1224-1229.

Worby, C. A., and J. E. Dixon. 2002. Sorting out the cellular functions of sorting nexins. Nat. Rev. Mol. Cell Biol. 3:919-931. 